\title{
INVERSE NODAL PROBLEMS FOR DIFFERENTIAL OPERATORS ON GRAPHS WITH A CYCLE
}

\author{
G. FREILING AND V. A. YURKO
}

\begin{abstract}
Inverse nodal problems are studied for second-order differential operators on graphs with a cycle and with standard matching conditions in the internal vertex. Uniqueness theorems are proved, and a constructive procedure for the solution is provided.
\end{abstract}

\section{Introduction}

The main purpose of this note is to study inverse nodal problems for Sturm-Liouville differential operators on graphs with a cycle. The inverse nodal problems under consideration consist in recovering operators from the given nodes (zeros) of eigenfunctions. These problems are related to some questions in mechanics and mathematical physics (see, for example, [1]). Moreover, there are close connections of this area with inverse spectral problems. Inverse nodal problems for Sturm-Liouville operators on an interval have been studied fairly completely in [1]-[6] and other papers. The main results on inverse spectral problems on an interval are presented in the monographs [7]-[14].

Differential operators on graphs (networks, trees) often appear in natural sciences and engineering (see [15], [16] and the references therein). In particular, inverse spectral problems of recovering coefficients of differential operators on graphs from their spectral characteristics were investigated in [17]- [23].

In this paper, we give the formulations and the solutions of inverse nodal problems for Sturm-Liouville operators on graphs with a cycle from given subsets of nodal points on a fixed edge or on a certain part of it. We prove the corresponding uniqueness theorems and provide a constructive procedure for the solution. We also show connections of these problems with inverse spectral problems on graphs.

Consider a compact graph $G$ in $\mathbf{R}^{\mathbf{m}}$ with the set of vertices $V=\left\{v_{0}, \ldots, v_{r}\right\}$ and the set of edges $\mathcal{E}=\left\{e_{0}, \ldots, e_{r}\right\}$, where $v_{1}, \ldots, v_{r}$ are the boundary vertices, $v_{0}$ is the internal vertex, $e_{j}=\left[v_{j}, v_{0}\right], j=\overline{1, r}, \bigcap_{j=0}^{r} e_{j}=\left\{v_{0}\right\}$, and $e_{0}$ is a cycle. Thus, the graph $G$ has one

Corresponding author: V. A. Yurko.

Received February 25, 2009.

2000 Mathematics Subject Classification. 34A55 34B24 47E05.

Key words and phrases. Sturm-Liouville operators, spatial networks, inverse nodal problems. 
cycle $e_{0}$ and one internal vertex $v_{0}$. We suppose that the length of each edge is equal to 1. Each edge $e_{j} \in \mathcal{E}$ is parameterized by the parameter $x \in[0,1]$; below we identify the value $x$ of the parameter with the corresponding point on the edge. It is convenient for us to choose the following orientation: for $j=\overline{1, r}$, the vertex $v_{j}$ corresponds to $x=0$, and the vertex $v_{0}$ corresponds to $x=1$; for $j=0$, both ends $x=+0$ and $x=1-0$ correspond to $v_{0}$.

An integrable function $Y$ on $G$ may be represented as $Y=\left\{y_{j}\right\}_{j=\overline{0, r}}$, where the function $y_{j}(x), x \in[0,1]$, is defined on the edge $e_{j}$. Let $q=\left\{q_{j}\right\}_{j=\overline{0, r}}$ be an integrable real-valued function on $G ; q$ is called the potential. Consider the following differential equation on $G$ :

$$
-y_{j}^{\prime \prime}(x)+q_{j}(x) y_{j}(x)=\lambda y_{j}(x), \quad j=\overline{0, r},
$$

where $\lambda$ is the spectral parameter, the functions $y_{j}, y_{j}^{\prime}, j=\overline{0, r}$, are absolutely continuous on $[0,1]$ and satisfy the following matching conditions in the internal vertex $v_{0}$ :

$$
\left.\begin{array}{c}
y_{j}(1)=y_{0}(0), \quad j=\overline{0, r} \quad \text { (continuity condition) }, \\
\sum_{j=0}^{r} y_{j}^{\prime}(1)=y_{0}^{\prime}(0) \quad \text { (Kirchhoff's condition). }
\end{array}\right\}
$$

The matching conditions (2) are called the standard conditions. In electrical circuits, (2) expresses Kirchhoff's law; in elastic string networks, it expresses the balance of tension, and so on.

Let us consider the boundary value problem $B=B(q)$ on $G$ for equation (1) with the matching conditions (2) and with Dirichlet boundary conditions at the boundary vertices $v_{1}, \ldots, v_{r}$ :

$$
y_{j}(0)=0, \quad j=\overline{1, r} .
$$

In section 2 we study the inverse nodal problem of recovering the potential from any dense subset of the nodal points of $B$. The uniqueness theorem is proved and a constructive procedure for the solution is provided. In section 3 we investigate the so-called incomplete inverse problems of recovering the potential on a fixed edge from a subset of nodal points situated only on a part of the edge. The main results of this section is presented in Theorem 4. In order to prove this theorem we use connections with inverse spectral problems established in Section 3. In particular, we essentially use the results on incomplete inverse spectral problem (presented in Theorem 3 ) of recovering the potential on a part of a fixed edge from a part of the spectrum of $B$.

We note that in the recent paper [24], inverse nodal problem on graphs was studied in a different formulation. It was proved in [24] that the specification of the spectrum and the set of all nodal points uniquely determines the potential. In the present paper we study a particular class of graphs with a cycle and show that we do not need to specify the spectrum. We pay our main attention to incomplete inverse spectral and nodal problems when nodal points are specified on a part of the graph. We also note that in [25] similar results were obtained on star-type graphs where the structure of the 
characteristic function is simpler than for graphs having a cycle; it turns out that the results of [25] also remain valid in the more general situation under consideration.

\section{Inverse nodal problems}

Denote by $S_{j}(x, \lambda)$ and $C_{j}(x, \lambda), j=\overline{0, r}$, the solutions of equation (1) on the edge $e_{i}$ satisfying the initial conditions

$$
S_{j}(0, \lambda)=C_{j}^{\prime}(0, \lambda)=0, \quad S_{j}^{\prime}(0, \lambda)=C_{j}(0, \lambda)=1 .
$$

For each fixed $x \in[0,1]$, the functions $S_{j}^{(\nu)}(x, \lambda)$ and $C_{j}^{(\nu)}(x, \lambda), j=\overline{1, r}, \nu=0,1$, are entire in $\lambda$ of order $1 / 2$. Moreover, the functions $S_{j}(x, \lambda)$ and $C_{j}(x, \lambda)$ are the unique solutions of the integral equations

$$
\begin{aligned}
& S_{j}(x, \lambda)=\frac{\sin \rho x}{\rho}+\int_{0}^{x} \frac{\sin \rho(x-t)}{\rho} q_{j}(t) S_{j}(t, \lambda) d t, \\
& C_{j}(x, \lambda)=\cos \rho x+\int_{0}^{x} \frac{\sin \rho(x-t)}{\rho} q_{j}(t) C_{j}(t, \lambda) d t,
\end{aligned}
$$

where $\lambda=\rho^{2}$. Using (4) and (5) one gets (see, for example, [10, Chap. 1] for details) the following asymptotical formulae as $|\lambda| \rightarrow \infty$ uniformly in $x \in[0,1]$ :

$$
\begin{aligned}
& S_{j}(x, \lambda)=\frac{\sin \rho x}{\rho}-\frac{\cos \rho x}{2 \rho^{2}} \int_{0}^{x} q_{j}(t) d t+\frac{1}{2 \rho^{2}} \int_{0}^{x} q_{j}(t) \cos \rho(x-2 t) d t+O\left(\frac{\exp (|\tau| x)}{\rho^{3}}\right), \\
& S_{j}^{\prime}(x, \lambda)=\cos \rho x+\frac{\sin \rho x}{2 \rho} \int_{0}^{x} q_{j}(t) d t-\frac{1}{2 \rho} \int_{0}^{x} q_{j}(t) \sin \rho(x-2 t) d t+O\left(\frac{\exp (|\tau| x)}{\rho^{2}}\right), \\
& C_{j}(x, \lambda)=\cos \rho x+\frac{\sin \rho x}{2 \rho} \int_{0}^{x} q_{j}(t) d t+\frac{1}{2 \rho} \int_{0}^{x} q_{j}(t) \sin \rho(x-2 t) d t+O\left(\frac{\exp (|\tau| x)}{\rho^{2}}\right) .
\end{aligned}
$$

where $\tau=\operatorname{Im} \rho$. Let us consider the function

$\Delta(\lambda)=\left(\prod_{j=1}^{r} S_{j}(1, \lambda)\right)\left(C_{0}(1, \lambda)+S_{0}^{\prime}(1, \lambda)-2\right)+\left(\sum_{j=1}^{r} S_{j}^{\prime}(1, \lambda) \prod_{k=1, k \neq j}^{r} S_{k}(1, \lambda)\right) S_{0}(1, \lambda)$.

The function $\Delta(\lambda)$ is entire in $\lambda$ of order $1 / 2$, and its zeros coincide with the eigenvalues of the boundary value problem $B$. Indeed, let

$$
\begin{aligned}
Y(x, \lambda) & =\left\{y_{j}(x, \lambda)\right\}_{j=\overline{0, r}} \\
y_{j}(x, \lambda) & =A_{j}(\lambda) S_{j}(x, \lambda), j=\overline{1, r}, \quad y_{0}(x, \lambda)=A_{0}(\lambda) S_{0}(x, \lambda)+B_{0}(\lambda) C_{0}(x, \lambda),
\end{aligned}
$$

where the functions $A_{j}(\lambda)$ and $B_{0}(\lambda)$ do not depend on $x$. Then the function $Y(x, \lambda)$ satisfies (1) and (3). Substituting (10) into (2) we obtain a linear homogeneous algebraic system $s$ with respect to $A_{j}(\lambda)$ and $B_{0}(\lambda)$. The determinant of this system is $\Delta(\lambda)$. If $\lambda_{0}$ is a zero of $\Delta(\lambda)$, then the function $Y\left(x, \lambda_{0}\right)$ of the form (10) is an eigenfunction, and $\lambda_{0}$ 
is an eigenvalue. Conversely, if $\lambda_{0}$ is an eigenvalue, then the corresponding eigenfunction has the form (10) with $\lambda=\lambda_{0}$. Since $Y \neq 0$, the algebraic system $s$ has a nontrivial solution, and consequently, $\Delta\left(\lambda_{0}\right)=0$. The function $\Delta(\lambda)$ is called the characteristic function for the boundary value problem $B$.

Substituting (6)-(8) into (9) we get

$$
\Delta(\lambda)=\Delta^{0}(\lambda)+\left(\frac{\sin \rho}{\rho}\right)^{r-1} \frac{F(\rho)}{\rho^{2}}+o\left(\frac{\exp ((r+1)|\tau|)}{\rho^{r+1}}\right), \quad|\lambda| \rightarrow \infty,
$$

where

$$
\begin{aligned}
F(\rho)= & \left(\int_{0}^{1} q_{0}(x) d x+\frac{1}{2} \int_{0}^{1} \sum_{j=1}^{r} q_{j}(x) d x\right) \sin ^{2} \rho \\
& -\left(\frac{r}{2} \int_{0}^{1} q_{0}(x) d x+\frac{r+1}{2} \int_{0}^{1} \sum_{j=1}^{r} q_{j}(x) d x\right) \cos ^{2} \rho+\left(\int_{0}^{1} \sum_{j=1}^{r} q_{j}(x) d x\right) \cos \rho, \\
\Delta^{0}(\lambda)= & \left(\frac{\sin \rho}{\rho}\right)^{r}((r+2) \cos \rho-2) .
\end{aligned}
$$

Notice that $\Delta^{0}(\lambda)$ is the characteristic function for the boundary value problem $B^{0}:=$ $B(0)$ with the zero potential. It follows from (13) that the boundary value problem $B^{0}$ has a countable set of eigenvalues $\Lambda^{0}=\left\{\lambda_{n s}^{0}\right\}_{n \geq 0, s=\overline{0, r}}$ (counting multiplicities), where $\lambda_{n s}^{0}=\left(\rho_{n s}^{0}\right)^{2}$,

$$
\begin{aligned}
\rho_{n s}^{0} & =(n+1) \pi, s=\overline{1, r}, \quad \rho_{n 0}^{0}=n \pi+\left(\frac{\pi}{2}+(-1)^{n}\left(\xi_{0}-\frac{\pi}{2}\right)\right), \\
\xi_{0} & =\arccos \frac{2}{r+2} \in\left(0, \frac{\pi}{2}\right) .
\end{aligned}
$$

Using (11) by the well-known method (see, for example, [10, Chap. 1]) we obtain that the boundary value problem $B$ has a countable set of eigenvalues $\Lambda=\left\{\lambda_{n s}\right\}_{n \geq 0, s=\overline{0, r}}$. All eigenvalues are real and have the asymptotics

$$
\rho_{n s}:=\sqrt{\lambda_{n s}}=\rho_{n s}^{0}+O\left(\frac{1}{n}\right), \quad s=\overline{0, r}, \quad n \rightarrow \infty,
$$

where $\rho_{n s}^{0}$ are calculated via (14) and (15).

Denote $\lambda_{n}:=\lambda_{n 0}, \rho_{n}:=\rho_{n 0}$. Substituting (16) into (11) and using (12) and the relation $\Delta\left(\lambda_{n}\right)=0$, we obtain the following more precise asymptotical formula

$$
\rho_{n}:=\sqrt{\lambda_{n}}=\rho_{n 0}^{0}+\frac{\xi_{1}}{\pi n}+o\left(\frac{1}{n}\right), \quad n \rightarrow \infty,
$$

where

$$
\xi_{1}=\frac{1}{r+4} \int_{0}^{1} q_{0}(x) d x+\frac{r+2}{2 r(r+4)} \int_{0}^{1} \sum_{j=1}^{r} q_{j}(x) d x .
$$


Using (6) and (17) we get the asymptotics for the components of the eigenfunctions as $n \rightarrow \infty$, uniformly in $x \in[0,1]$ :

$$
\rho_{n} S_{i}\left(x, \lambda_{n}\right)=\sin \rho_{n 0}^{0} x-\frac{1}{2 \pi n}\left(\int_{0}^{x} q_{i}(x) d x-2 \xi_{1} x\right) \cos \rho_{n 0}^{0} x+o\left(\frac{1}{n}\right),
$$

where $\rho_{n 0}^{0}$ and $\xi_{1}$ are calculated by (14) and (18), respectively.

Fix $i=\overline{1, r}$. There exists $N_{0}$ such that for all $n>N_{0}$ the function $S_{i}\left(x, \lambda_{n}\right)$ has exactly $n$ (simple) zeros inside the interval $(0,1)$, namely: $0<x_{n i}^{1}<\cdots<x_{n i}^{n}<1$. The points $X_{i}:=\left\{x_{n i}^{j}\right\}$ are called nodal points on the edge $e_{i}$ related to the eigenvalues $\left\{\lambda_{n}\right\}$.

Fix $i=\overline{1, r}$. We will consider the inverse nodal problem of recovering the potential $q_{i}(x)$ on the edge $e_{i}$ from the given set $X_{i}$ of nodal points or from a certain of it part. Denote

$$
\alpha_{n}^{j}:=\frac{\pi j}{\rho_{n 0}^{0}} \quad n \geq 1, \quad j=\overline{1, n} .
$$

Clearly, if $q=0$, then $x_{n i}^{j}=\alpha_{n}^{j}$ for all $n \geq 1, i=\overline{1, r}, j=\overline{1, n}$.

Taking (19) into account, we obtain the following asymptotic formula for the nodal points as $n \rightarrow \infty$, uniformly in $j$ :

$$
x_{n i}^{j}=\alpha_{n}^{j}+\frac{1}{\pi n^{2}}\left(\int_{0}^{\alpha_{n}^{j}} q_{i}(t) d t-2 \xi_{1} \alpha_{n}^{j}\right)+o\left(\frac{1}{n^{2}}\right),
$$

where the numbers $\alpha_{n}^{j}$ are defined by (20). We note that for each fixed $i=\overline{1, r}$, the set $X_{i}$ is dense on $(0,1)$. Without loss of generality we assume that $\xi_{1}=0$ (this can be achieved by the shift: $\left.q_{i}(x) \rightarrow q_{i}(x)-2 \xi_{1}, \lambda \rightarrow \lambda-2 \xi_{1}\right)$. Using (21) we arrive at the following assertion.

Theorem 1. Fix $i=\overline{1, r}$ and $x \in[0,1]$. Let $X_{i}^{0} \subset X_{i}$ be dense on $(0,1)$. Let $\left\{x_{n i}^{j_{n i}}\right\} \in X_{i}^{0}$ be chosen such that $\lim _{n \rightarrow \infty} x_{n i}^{j_{n i}}=x$. Then there exists a finite limit

$$
g_{i}(x):=\lim _{n \rightarrow \infty} \pi n^{2}\left(x_{n i}^{j_{n i}}-\alpha_{n}^{j_{n i}}\right),
$$

and

$$
g_{i}(x)=\int_{0}^{x} q_{i}(t) d t .
$$

Let us now formulate an uniqueness theorem and provide a constructive procedure for the solution of the inverse nodal problem. For this purpose, together with $B$ we consider a boundary value problem $\tilde{B}=B(\tilde{q})$ of the same form but with a different potential $\tilde{q}$. We agree that if a certain symbol $\alpha$ denotes an object related to $B$, then $\tilde{\alpha}$ will denote an analogous object related to $\tilde{B}$.

Theorem 2. Fix $i=\overline{1, r}$. Let $X_{i}^{0} \subset X_{i}$ be a subset of the nodal points which is dense on $(0,1)$. Let $X_{i}^{0}=\tilde{X}_{i}^{0}$. Then $q_{i}(x)=\tilde{q}_{i}(x)$ a.e. on $(0,1)$. Thus, the specification of $X_{i}^{0}$ uniquely determines the potential $q_{i}(x)$ on the edge $e_{i}$. The function $q_{i}(x)$ can be constructed via the formula

$$
q_{i}(x)=g_{i}^{\prime}(x),
$$

where $g_{i}(x)$ is calculated by $(22)$. 
Indeed, formula (24) follows from (23). If $X_{i}^{0}=\tilde{X}_{i}^{0}$, then (22) yields $g_{i}(x) \equiv$ $\tilde{g}_{i}(x), x \in[0,1]$, and consequently, $q_{i}(x)=\tilde{q}_{i}(x)$ a.e. on $(0,1)$.

\section{Incomplete inverse problems}

First we consider the following incomplete inverse spectral problem. Fix $i, i=\overline{1, r}$. Suppose that $q_{k}(x)$ are known a priori for $k=\overline{0, r} \backslash i, x \in(0,1)$. Moreover, suppose that $q_{i}(x)$ is known on a part of the interval, namely, for $x \in(b, 1)$. The inverse problem is to construct $q_{i}(x)$ for $x \in(0, b)$ from a part of the spectrum of the boundary value problem $B$. Denote by $\sigma_{k}$ the spectrum of the boundary value problem

$$
-y_{k}^{\prime \prime}+q_{k}(x) y_{k}=\lambda y_{k}, \quad y_{k}(0)=y_{k}(1)=0 .
$$

Theorem 3. Fix $i, i=\overline{1, r}$ and $b \in(0,1 / 2)$. Let $q_{k}(x)=\tilde{q}_{k}(x)$ for $k=\overline{0, r} \backslash i$, a.e. on $(0,1)$, and $q_{i}(x)=\tilde{q}_{i}(x)$ a.e. on $(b, 1)$. Let $M \subset \mathbf{N} \cup\{0\}$ be a subset of the nonnegative integer numbers, and let $\Omega:=\left\{\lambda_{n}\right\}_{n \in M}$ be a part of the spectrum of $B$ such that $\sigma_{k} \cap \Omega=\emptyset, k=\overline{0, r} \backslash i$, and the system of functions $\left\{\cos 2 \rho_{n} x\right\}_{n \in M}$ is complete in $L_{2}(0, b)$. If $\Omega=\tilde{\Omega}$, then $q_{i}(x)=\tilde{q}_{i}(x)$ a.e. on $(0,1)$.

Proof. Since $\sigma_{k} \cap \Omega=\emptyset, k=\overline{0, r} \backslash i$, one has

$$
S_{k}\left(1, \lambda_{n}\right) \neq 0, \quad k=\overline{0, r} \backslash i, \quad \lambda_{n} \in \Omega .
$$

Let $Y_{n}(x)=\left\{y_{j n}(x)\right\}_{j=\overline{0, r}}$ be an eigenfunction related to the eigenvalue $\lambda_{n}$. Then

$$
y_{j n}(x)=A_{j n} S_{j}\left(x, \lambda_{n}\right), j=\overline{1, r}, \quad y_{0 n}(x)=A_{0 n} S_{0}\left(x, \lambda_{n}\right)+B_{0 n} C_{0}\left(x, \lambda_{n}\right),
$$

where $A_{j n}$ and $B_{0 n}$ are constants. Since $Y_{n}$ is not identically zero and satisfies the matching conditions (2), one has $A_{j n} \neq 0$ for all $j=\overline{1, r}$. Therefore, in view of (25), $y_{j n}(1) \neq 0$ for all $j=\overline{0, r}$, and $y_{0 n}(0) \neq 0$. Using the matching conditions (2) again we obtain

$$
\frac{y_{0 n}^{\prime}(0)}{y_{0 n}(0)}=\frac{y_{0 n}^{\prime}(1)}{y_{0 n}(1)}+\sum_{j=1}^{r} \frac{S_{j}^{\prime}\left(1, \lambda_{n}\right)}{S_{j}\left(1, \lambda_{n}\right)} .
$$

Without loss of generality we put $y_{0 n}(0)=1$. Then, in view of $(2), y_{j n}(1)=1, j=\overline{0, r}$. It follows from (26) that

$$
B_{0 n}=1, \quad A_{0 n}=\frac{1-C_{0}\left(1, \lambda_{n}\right)}{S_{0}\left(1, \lambda_{n}\right)}, \quad A_{j n}=\frac{1}{S_{j}\left(1, \lambda_{n}\right)}, j=\overline{1, r} .
$$

Since $q_{k}=\tilde{q}_{k}$ for $k=\overline{0, r} \backslash i$, one has

$$
S_{k}(x, \lambda) \equiv \tilde{S}_{k}(x, \lambda), \quad C_{k}(x, \lambda) \equiv \tilde{C}_{k}(x, \lambda), \quad k=\overline{0, r} \backslash i, \quad x \in[0,1],
$$

and consequently,

$$
A_{j n}=\tilde{A}_{j n}, \quad j=\overline{0, r} \backslash i .
$$


Together with (26) this yields

$$
y_{0 n}(x) \equiv \tilde{y}_{0 n}(x)
$$

Taking (27) into account we infer

$$
\frac{S_{i}^{\prime}\left(1, \lambda_{n}\right)}{S_{i}\left(1, \lambda_{n}\right)}=\frac{\tilde{S}_{i}^{\prime}\left(1, \lambda_{n}\right)}{\tilde{S}_{i}\left(1, \lambda_{n}\right)}, \quad \lambda_{n}=\tilde{\lambda}_{n} \in \Omega
$$

Since

$$
\begin{gathered}
-S_{i}^{\prime \prime}(x, \lambda)+q_{i}(x) S_{i}(x, \lambda)=\lambda S_{i}(x, \lambda), \quad-\tilde{S}_{i}^{\prime \prime}(x, \lambda)+\tilde{q}_{i}(x) \tilde{S}_{i}(x, \lambda)=\lambda \tilde{S}_{i}(x, \lambda), \\
S_{i}(0, \lambda)=\tilde{S}_{i}(0, \lambda)=0, \quad S_{i}^{\prime}(0, \lambda)=\tilde{S}_{i}^{\prime}(0, \lambda)=1,
\end{gathered}
$$

it follows that

$$
\int_{0}^{1} Q_{i}(x) S_{i}(x, \lambda) \tilde{S}_{i}(x, \lambda) d x \equiv S_{i}^{\prime}(1, \lambda) \tilde{S}_{i}(1, \lambda)-S_{i}(1, \lambda) \tilde{S}_{i}^{\prime}(1, \lambda),
$$

where $Q_{i}(x)=q_{i}(x)-\tilde{q}_{i}(x)$. Using (28) we calculate

$$
\int_{0}^{b} Q_{i}(x) S_{i}\left(x, \lambda_{n}\right) \tilde{S}_{i}\left(x, \tilde{\lambda}_{n}\right) d x=0, \quad \lambda_{n}=\tilde{\lambda}_{n} \in \Omega .
$$

It is known (see $[7,8,10])$ that the following representation holds

$$
S_{i}(x, \lambda)=\frac{\sin \rho x}{\rho}+\int_{0}^{x} K_{i}(x, t) \frac{\sin \rho t}{\rho} d t,
$$

where $K_{i}(x, t)$ is a smooth function which does not depend on $\lambda$. By virtue of (31) one gets

$$
2 \rho^{2} S_{i}(x, \lambda) \tilde{S}_{i}(x, \lambda)=1-\cos 2 \rho x-\int_{0}^{x} V_{i}(x, t) \cos 2 \rho t d t,
$$

where $V_{i}(x, t)$ is a continuous function which does not depend on $\lambda$. Substituting (32) into (30) and taking the relation $\int_{0}^{b} Q_{i}(x) d x=0$ into account, we calculate

$$
\int_{0}^{b}\left(Q_{i}(x)+\int_{x}^{b} V_{i}(t, x) Q_{i}(t) d t\right) \cos 2 \rho_{n} x d x=0, \quad \lambda_{n} \in \Omega,
$$

and consequently,

$$
Q_{i}(x)+\int_{x}^{b} V_{i}(t, x) Q_{i}(t) d t=0 \quad \text { a.e. on } \quad(0, b) .
$$

Since this homogeneous integral equation has only the trivial solution it follows that $Q_{i}(x)=0$ a.e. on $(0, b)$, i.e. $q_{i}(x)=\tilde{q}_{i}(x)$ a.e. on $(0, b)$.

Let us go on to the investigation of an incomplete inverse nodal problem when nodal points are given only on a part of the interval. First we recall for convenience of the reader an auxiliary assertion (see [5], [6]) including a short proof. 
Lemma 1. Fix $n, j, i$. Let $x_{n i}^{j}=\tilde{x}_{n i}^{j}, x_{n i}^{j+1}=\tilde{x}_{n i}^{j+1}$, and let $q_{i}(x)=\tilde{q}_{i}(x)$ a.e. on $\left(x_{n i}^{j}, x_{n i}^{j+1}\right)$. Then $\lambda_{n}=\tilde{\lambda}_{n}$.

Proof. On the interval $x \in\left(x_{n i}^{j}, x_{n i}^{j+1}\right)$ we consider the boundary value problem $B_{n i}^{j}$ for equation (1) with the boundary conditions

$$
y\left(x_{n i}^{j}\right)=y\left(x_{n i}^{j+1}\right)=0 .
$$

The function $y_{n i}(x)=S_{i}\left(x, \lambda_{n}\right)$ is the eigenfunction of $B_{n i}^{j}$ related to the eigenvalue $\lambda_{n}$. Since $y_{n i}(x)$ has no zeros for $x \in\left(x_{n i}^{j}, x_{n i}^{j+1}\right)$, it follows from Sturm's oscillation theorem that $\lambda_{n}$ is the first eigenvalue of $B_{n i}^{j}$, and $y_{n i}(x)$ is the first eigenfunction. Since $q_{i}(x)=\tilde{q}_{i}(x)$ a.e. on $\left(x_{n i}^{j}, x_{n i}^{j+1}\right)$, one has $\lambda_{n}=\tilde{\lambda}_{n}$.

For $X \subset X_{i}$ we denote $\Lambda_{X}:=\left\{n: \exists j x_{n i}^{j} \in X\right\}$.

Definition 1. Let $X \subset X_{i}$. The set $X$ is called twin if together with each of its points $x_{n i}^{j}$ the set $X$ contains at least one of the adjacent nodal points $x_{n i}^{j-1}$ or $x_{n i}^{j+1}$.

Theorem 4. Fix $i=\overline{1, r}$ and $b \in(0,1 / 2)$. Let $q_{k}(x)=\tilde{q}_{k}(x)$ for $k=\overline{1, r} \backslash i$, a.e. on $(0,1)$. Let $X \subset X_{i} \cap(b, 1)$ be a dense on $(b, 1)$ twin subset of nodal points such that $\sigma_{k} \cap \Omega_{\Lambda_{X}}=\emptyset, k=\overline{1, r} \backslash i$, and the system of functions $\left\{\cos 2 \rho_{n} x\right\}_{n \in \Lambda_{X}}$ is complete in $L_{2}(0, b)$. If $X=\tilde{X}$, then $q_{i}(x)=\tilde{q}_{i}(x)$ a.e. on $(0,1)$.

Thus, the specification of nodal point on a part of the interval uniquely determines $q_{i}(x)$ on the whole interval $(0,1)$.

Proof. Since $X=\tilde{X}$, it follows that $g_{i}(x) \equiv \tilde{g}_{i}(x)$ for $x \in(b, 1)$. Using (23) we obtain $q_{i}(x)=\tilde{q}_{i}(x)$ a.e. on $(b, 1)$. By Lemma $1, \lambda_{n}=\tilde{\lambda}_{n}$ for $n \in \Lambda_{X}$. Applying Theorem 3 we conclude that $q_{i}(x)=\tilde{q}_{i}(x)$ a.e. on $(0,1)$.

\section{Acknowledgement}

This research was supported in part by DAAD and Grants 07-01-00003 and 07-0192000-NSC of Russian Foundation for Basic Research.

\section{References}

[1] J. R. McLaughlin, Inverse spectral theory using nodal points as data - a uniqueness result, J. Differ. Equat. 73 (1988), 354-362.

[2] C. L. Shen and T. M. Tsai, On a uniform approximation of the density function of a string equation using EVs and nodal points and some related inverse nodal problems, Inverse Problems 11 (1995), 1113-1123.

[3] C. K. Law and C. F. Yang, Reconstructing the potential function and its derivatives using nodal data, Inverse Problems 14 (1998), 299-312. 
[4] C. L. Shen and C. T. Shieh, An inverse nodal problem for vectorial Sturm-Liouville equation, Inverse Problems 16 (2000), 349-56.

[5] X. F. Yang, A new inverse nodal problem, J. Diff. Equations 169 (2001), 633-653.

[6] Y. H. Cheng, C. K. Law and J. Tsay, Remarks on a new inverse nodal problem, J. Math. Anal. Appl. 248 (2000), 145-155.

[7] V. A. Marchenko, Sturm-Liouville Operators and their Applications, Naukova Dumka, Kiev, 1977; English transl., Birkhäuser, 1986.

[8] B. M. Levitan, Inverse Sturm-Liouville Problems, Nauka, Moscow, 1984; English transl., VNU Sci.Press, Utrecht, 1987.

[9] J. Pöschel and E. Trubowitz, Inverse Spectral Theory. New York, Academic Press, 1987.

[10] G. Freiling and V. A. Yurko, Inverse Sturm-Liouville Problems and their Applications. NOVA Science Publishers, New York, 2001.

[11] V. A. Yurko, Method of Spectral Mappings in the Inverse Problem Theory, Inverse and Ill-posed Problems Series. VSP, Utrecht, 2002.

[12] K. Chadan, D. Colton, L. Paivarinta and Rundell W., An introduction to inverse scattering and inverse spectral problems. SIAM Monographs on Mathematical Modelling and Computation. SIAM, Philadelphia, PA, 1997.

[13] R. Beals, P. Deift and C. Tomei, Direct and Inverse Scattering on the Line, Math. Surveys and Monographs, v.28. Amer. Math. Soc. Providence: RI, 1988.

[14] V. A. Yurko, Inverse Spectral Problems for Differential Operators and their Applications. Gordon and Breach, Amsterdam, 2000.

[15] Yu. Pokornyi and A. Borovskikh, Differential equations on networks (geometric graphs), J. Math. Sci. (N.Y.) 119 (2004), 691-718.

[16] Yu. Pokornyi and V. Pryadiev, The qualitative Sturm-Liouville theory on spatial networks, J. Math. Sci. (N.Y.) 119 (2004), 788-835.

[17] N. I. Gerasimenko, Inverse scattering problem on a noncompact graph, Teoret. Mat. Fiz. 74 (1988), 187-200 (Russian); English transl. in Theor. Math. Phys. 75 (1988), 460-470.

[18] M. I. Belishev, Boundary spectral inverse problem on a class of graphs (trees) by the BC method, Inverse Problems 20 (2004), 647-672.

[19] V. A. Yurko, Inverse spectral problems for Sturm-Liouville operators on graphs, Inverse Problems 21 (2005), 1075-1086.

[20] B. M. Brownand R. Weikard, A Borg-Levinson theorem for trees, Proc. R. Soc. Lond. Ser. A Math. Phys. Eng. Sci. 461(2005), 3231-3243.

[21] V. A. Yurko, Reconstruction of higher-order differential operators on compact graphs, Doklady Akad. Nauk 419, no.5 (2008), 604-608; English transl: Doklady Mathematics 77(2008), 293-297.

[22] G. Freiling and V. A. Yurko, Inverse spectral problems for Sturm-Liouville operators on noncompact trees, Results in Math. 50 (2007), 195-212.

[23] V. A. Yurko, Inverse problems for Sturm-Liouville operators on graphs with a cycle, Operators and Matrices 2 (2008), 543-553.

[24] B. A. Watson and S. Currie, Inverse nodal problems for Sturm-Liouville equations on graphs, Inverse Problems 23 (2007), 2029-2040.

[25] V. A. Yurko, Inverse nodal and inverse spectral problems for differential operators on graphs, Journal of Inverse and Ill-Posed Problems 16 (2008), 715-722. 
Department of Mathematics, Duisburg-Essen University, Campus Duisburg, Forsthausweg 2, D-47057, Duisburg, Germany.

E-mail: gerhard.freiling@uni-due.de

Department of Mathematics, Saratov University, Astrakhanskaya 83, Saratov 410026, Russia.

E-mail: yurkova@info.sgu.ru 